\title{
THE GALACTIC DYNAMO: MODES AND MODELS
}

\author{
G. RÜDIGER, D. ELSTNER, M. SCHULTZ \\ Astrophysikalisches Institut Potsdam \\ An der Sternwarte 16, D-O-1590 Potsdam, Germany
}

\begin{abstract}
The hitherto known essentials of the present-day dynamo theory of the galactic magnetic fields are reported. The vertical stratification of the interstellar turbulence is exclusively considered as the source of the $\alpha$-effect. New expressions for this tensorial effect and its quenching by the field are applied. Although its anisotropy and the influence of the halo easily yield the excitation of non-axisymmetric magnetic configurations of diverse equatorial symmetry, the galactic differential rotation finally leads to axisymmetric and quadrupolar solutions. The magnetic strength of the dynamo fields slightly exceeds the equipartition value while its angular momentum transport (into the intergalactic space) is very small.

For increasingly steep vertical gradients of the turbulence intensity a saturation of the field strength is found since the eddy diffusivity grows with the $\alpha$-effect. Only beyond the maximum of the field the equatorial symmetry changes to an equatorial antisymmetry, i.e. to dipolar solutions.

In order to produce the observed values of the pitch angles one has only to choose correlation times of the turbulence slightly exceeding the usually accepted $10^{7} \mathrm{yrs}$.
\end{abstract}

\section{Introduction}

This report intends to continue the article "Key problems of flat object dynamo theory and ways to their solution" given in the Proceedings of the IAU symposium Nr. 140 (Krause et al. 1990). We want to answer, in particular, the old key question (non-axisymmetric modes in axisymmetric configurations?) and we shall formulate some new ones such as: i) how strong are the fields? ii) how much angular momentum do they transport? and iii) what is the field geometry?

Our answers have been obtained with a computer code for models "without sharp boundaries" (Stepinski \& Levi 1988, Elstner et al. 1990). The first relevant findings for "flat" models have been obtained applying this code for linear, kinematic dynamos (Meinel et al. 1990, Elstner et al. 1992). It was found that the galactic differential rotation always prevents the excitation of non-axisymmetric magnetic field configurations. Such a magnetic "BSS" structure is observed in one case (M81, M.Krause et al. 1989), but its coexistence with differential rotation is hard to understand. A detailed analyses of the interaction of anisotropic $\alpha$-effect and differential rotation in stellar cases has recently been presented by Rüdiger \& Elstner (1993).

A few years ago, the standard procedure was to form the $\alpha$-effect with the observed characteristics of giant molecular clouds, hence $\alpha \leq 5 \mathrm{~km} / \mathrm{s}$ and eddy diffusivity $\eta_{T} \leq 10^{26} \mathrm{~cm}^{2} / \mathrm{s}$ so that the dynamo number $C_{\alpha}=\alpha H / \eta_{T} \approx 1-10$ with $\mathrm{H} \simeq 1 \mathrm{kpc}$ as the thickness of the galaxy: enough for dynamo excitation. Only density stratification was considered as the source of the $\alpha$-effect, its anisotropy was ignored. On the other hand, Rüdiger (1990) and Ferrière (1992) have started to derive the $\alpha$-tensor as due to the action of random supernova (SN) explosions. The turbulence in galaxies has been replaced by the explosion flow pattern of a field of random SN explosions under the common influence of global rotation and a largescale magnetic field. This concept allowed the unified derivation of the complete turbulent EMF, $\mathcal{E}=\left\langle\mathbf{u}^{\prime} \times \mathbf{B}^{\prime}\right\rangle$, on the basis of one and the same turbulence flow 
field. For the first time in the history of the mean-field dynamo theory the series expansion

$$
\mathcal{E}_{i}=\alpha_{i j} \bar{B}_{i}-\eta_{i j k} \bar{B}_{k, j}+\ldots
$$

became fully tractable. Considering that the internal rotation law in galaxies is also (empirically) known, the observation of large-scale magnetic fields in galaxics (Sofue et al. 1988) opened a very promising chapter in the theory of the turbulent dynamo.

\section{Alpha and alpha-quenching}

The $\alpha$-effect only exists in a distributed turbulent rotating medium. Density stratification as well as intensity stratification of the turbulence ficld are known as $\alpha$ sources. In contrast to stellar convection zones the galactic disks are sclf-gravitating layers hence there arc only smooth density stratifications. It is thus not trivial to compute the $\alpha$-efrect numerically but in any case it makes no sense simply to identify turbulence with the $\alpha$-effect.

Ferrière (1992, also these proceedings) proposed to locate SN explosions at the galactic midplane ignoring the density stratification. This is confirmed by numerical simulations of SN explosions where the stratification only plays a minor role (Kaisig et al. 1993, also these proccedings). In both approaches the medium has been considered as perfect conductor, subject to the global galactic rotation and a homogeneous, large-scale magnetic ficld. The resulting EMF is represented by the relatively small numerical values

$$
|\alpha| \simeq 6 \cdot 10^{3} \mathrm{~cm} / \mathrm{s}, \quad \eta_{T} \simeq 5 \cdot 10^{24} \mathrm{~cm}^{2} / \mathrm{s},
$$

so that the normalized $\alpha$ for $\mathrm{SN}$ explosions results in

$$
C_{\alpha} \simeq 5
$$

As the value (3) is sufficiently large, it is thus shown that at least $\alpha^{2}$-dynamos can cxist in galaxies by the action of random $S N$ cxplosions. With a special flow ficld of astrophysical relevance it is so firstly shown that in galactic dimensions large-scale magnetic fields can be originated. Due to the low value of the eddy diffusivity, however, the influence of the differential rotation in such dynamos would be extremely strong. Very small pitch angles were the immediate consequence, in contradiction to the observations.

The $\alpha$-effect is a tensor ( $c$. Stecnbeck clal. 1966, Moffatt 1970, Roberts \& Soward 1975, Wälder ct al. 1980, Rüdiger 1980). It was striking that for the vertical $\alpha$-cffect (which acts on $B_{z}$ ) ncgative values appcared on the northern hemisphere (and positive at the southern hemisphere) in opposition to the cxpected positive (negative) values. Also numerical simulations of convection in unstable temperature stratifications led to the same result (Brandenburg ct al. 1990). Obviously, the anisotropy of the $\alpha$-tensor is so strong that it leads to different signs in different directions. The $\alpha$-tensor for slow rotation has the simple form

$$
\alpha_{i j}=\alpha_{1}(\mathrm{U} \Omega) \delta_{i j}+\alpha_{2}\left(U_{i} \Omega_{j}+U_{j} \Omega_{i}\right)+\ldots
$$


with $\mathbf{U}=\nabla \log \sqrt{\left\langle u^{\prime 2}\right\rangle}$. The existing literature of the mean-field dynamo deals only with the application of the first coefficient, i.c. for isotropic $\alpha$. We will demonstrate, however, that it will be dominated by terms ignored so far.

A re-discussion for the $\alpha$-effect in mean-field theory is thus in order. A turbulence field involving stratification was subjected to a global rotation. The resulting turbulence field under the influence of the large-scale magnetic ficld produces magnetic fluctuations which correlate with the velocity itself. While the rotation has been assumed as slow (which is true in galaxies) the magnetic ficld is of arbitrary strength. Its non-linear influence represents the back-rcaction of the magnetic field on the turbulence (Rüdiger \& Kichatinov 1993).

Our turbulcnce model has special properties: i) the anclastic approximation is adopted, ii) the cells possess a characteristic sizc, $l_{\text {corr }}$, and iii) a corrclation time, $\tau_{\text {corr }}$, is introduced only via a background eddy viscosity, $\nu_{\iota}=\eta_{l}$ :

$$
\tau_{\text {corr }}=\frac{l_{\text {corr }}^{2}}{\nu_{t}} \simeq l_{\text {corr }} / u^{\prime}
$$

(" $\tau$-approximation", Kichatinov 1987, 1991). Additionally, of course, the quasilincar approximation is used to derive the $\alpha$-tcnsor, which we developed for the case of uniform density. Recall that numerical simulations of SN-cxplosions revealed, indeed, that the influence of density stratification is extrcmcly small. The resulting expressions are

$$
\begin{array}{r}
\alpha_{r r}=\alpha_{\phi \phi}=-\frac{4}{15} \tau_{\text {corr }}^{2} \frac{d<u^{\prime 2}>}{d z}\left(\psi+\frac{15}{8} \frac{B_{z}^{2}}{B^{2}} \psi_{1}\right) \Omega \\
\alpha_{z z}=\frac{8}{15} \tau_{c o r r}^{2} \frac{d<u^{\prime 2}>}{d z}\left(\psi_{z}-\frac{15}{16} \frac{B_{z}^{2}}{B^{2}} \psi_{1}\right) \Omega \\
\alpha_{r \phi}=-\alpha_{\phi r}=\frac{1}{6} \tau_{c o r r} \frac{d<u^{\prime 2}>}{d z} \psi_{d i a}
\end{array}
$$

(in cylindrical polar coordinates, the $\psi$ 's arc normalized to unity for $B \rightarrow 0$ ). As usual the ratio of the diagonal terms and the off-diagonal terms of the $\alpha$-tensor strongly depends on the rotational rate, $\Omega$, or the turnover time $\tau_{c o r r}$, resp. For too short turnover times, e.g., the advection terms can really dominate. The dynamo cannot survive a too dominating advection. The quenching functions $\psi$, which at present are only known for the given stratification $U$ can be found in Rüdiger \& Kichatinov (1993).

One can take from Eqs. (6) that the vertical $\alpha, \alpha_{z i}$, has the opposite sign as the horizontal one. The consequences of this behaviour are presented in the following section.

With respect to the eddy diffusivity, we are still forced to work with the traditional scalar expression

$$
\eta_{r}=c_{\eta}<u^{\prime 2}>\tau_{\text {corr }}, \quad c_{\eta} \simeq 1 / 3
$$

which, for slow rotation, should not be too wrong. For rapid rotation and strong magnetic fields, of course, the scalar quantity $\eta_{T}$, will loose its sense and a tensor 

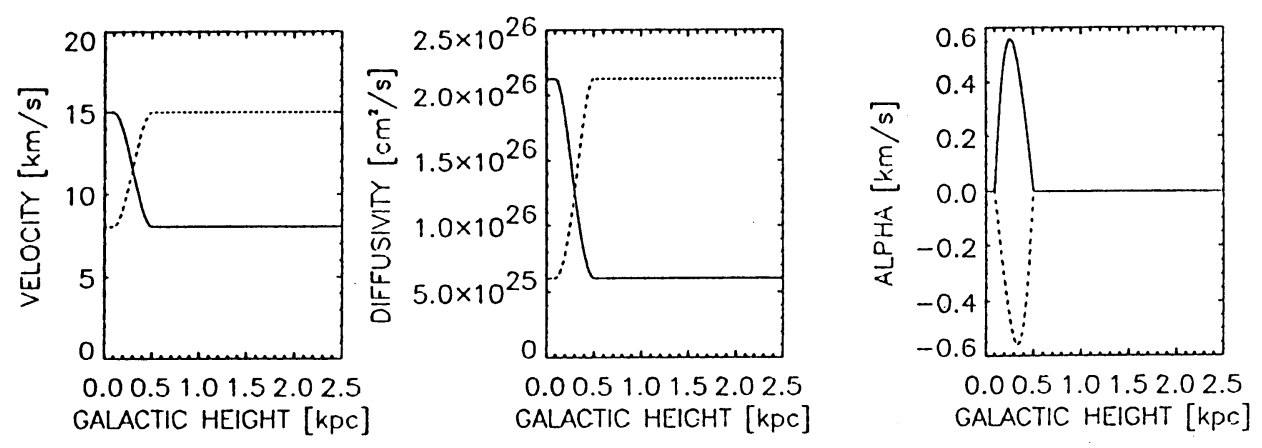

Fig. 1. Eddy diffusivity and horizontal $\alpha$-cffect for two differcnt $\%$-profiles of the turbulcnec velocity. Density stratification is excluded. Note the different sigus of the $\alpha$-ceffect for the diffcrent profilcs

appears. Magnetic quenching of the diffusivity tensor is not considered in galactic dynamos so far. On the other hand, the exclusive involution of the magnetic feedback in only one part of the turbulent EMF is of a very restricted valuc.

In Fig. 1 the eddy diffusivity and the horizontal $\alpha$-effect, $\alpha^{\prime} \phi \psi$, for a model with uniform density and diverse profiles of the turbulence intensity are given. The first one describes a turbulence field with an rms intensity of $10 \mathrm{~km} / \mathrm{s}$ reducing to $5 \mathrm{~km} / \mathrm{s}$ in a height of $500 \mathrm{pc}$. The horizontal $\alpha$ pealis in the middle of this region, is positive and vanishes outside. The eddy diffusivity (7) is large around the midplane and is reduced outside by a factor of 4 . Throughout the present report we are working with a uniform correlation time of the eddics of $\tau_{\text {corr }} \gtrsim 10^{7}$ yrs ( $\sim$ lifetime of giant molccular clouds).

There are suggestions that the turbulcnt velocity may grow with increasing: galactic heights (Brandenburg clal. 1993). Without the density stratification, the related $\alpha$-cflect proved to be negative. 'The density stratification is probably not strong enough to overcompensate this bchaviour. With the typical density stratification,

$$
\rho=\frac{4 \rho_{c}}{\left(c^{\frac{22}{\pi}}+c^{-\frac{22}{\pi}}\right)^{2}}
$$

included, we obtain zero, or negative values for the $\alpha$ unless the scalc of the turbulence intensity is very large. When normalized with the eddy diflusivity, we observe the typical picture of a "halo-dynamo" with very negalive $\alpha$ in the disk and positive $\alpha$ in the outer regions ( $c$. Fig. 2). Also the "cscapc velocity" $\alpha_{\psi r}$. changes its sign and turns toward the midplane. There is certainly more work needed lo formulate the interesting interplay betwecn $\alpha$-eflect, density stratification and the distribution of the turbulence intensity. 


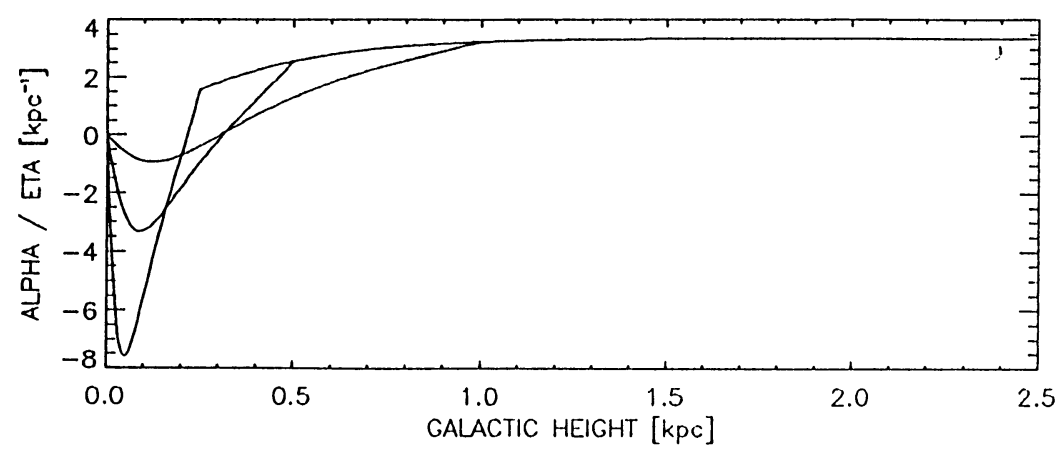

Fig. 2. Horizontal $\alpha$-effect divided by the eddy diffusivity for turbulcnce intcnsitics growing with galactic height $\left(u_{i n}=10 \mathrm{~km} / \mathrm{s}, u_{o u t}=100 \mathrm{~km} / \mathrm{s}\right.$, density scalc height $\left.500 \mathrm{pc}\right)$. The $\alpha$-effect in the galactic disks tends to vanish or becomes even negative

\section{Modes and models}

In this section we will examine the kinematic dynamo theory for disk configurations. The disks are defined by corresponding profiles of the turbulence parameters:

$$
\alpha=\alpha(r, z), \quad \eta_{T}=\eta_{T}(r, z) .
$$

Strict vacuum does not exist. The $\alpha$-layer is cmbedded in a medium of prescribed conductivity. The aspect ratio of the disk is chosen as 1:10. In terms of the stability analysis of Krause \& Meinel (1988) only the magnetic modes with the lowest eigenvalues for $C_{\alpha}$ are discussed, (Tab. I). We observe two important results: i) anisotropic $\alpha$ clearly favours non-axisymmetric modes with the azimuthal "quantum number" $m=1$ and ii) the "halo" conductivity plays a big part. The corners of Tab. I are occupied by four completely different magnetic configurations.

The S0 mode plays a particularly important rolc. It bifurcates first in the majority of models with isotropic $\alpha$ if the outer conductivity is not too small ( $c f$. Moss \& Brandenburg 1992). Dipolar solutions only occur for weak differential rotation and/or $\alpha$-effect with special radial profiles (see below).

One could believe that there are no longer difficulties in fitting the observations to the physical conditions described in Tab. I. The existence of BSS modes in M81 gives strong evidence for the existence of the anisotropic $\alpha$-tensor. For example, if non-axisymmetric quadrupoles were observed then the outer medium cannot be a hot plasma. However, the strong action of the differential rotation disturbs the simple picture. As known, beyond a rigidly rotating" core" with $r=r_{\Omega}$, the rotation rate is no longer uniform:

$$
\Omega=\Omega_{0}\left\{\begin{array}{ll}
1 & \text { if } r<r_{\Omega} \\
r_{\Omega} / r & \text { if } r>r_{\Omega}
\end{array} .\right.
$$

The well-defined uniform linear velocity of the outer galactic part is $V=r_{\Omega} \Omega_{0}$. If we leave it constant for a set of models (c.g. $200 \mathrm{~km} / \mathrm{s}$ ) then the rotation rate of the 
TABLE I

The leading modes for $\alpha^{2}$-disk dynamos, i.c. without differcntial rotation. The $\alpha$-effect can be anisotropic, the halo has divcrsc elcctrical features. If morc than 1 mode is given in a box then the eigenvalucs lie very close together

\begin{tabular}{c|ccc}
\hline disk embedded in & $\alpha_{z z}=\alpha_{\phi \phi}$ & $\alpha_{z z}=0$ & $\alpha_{z z}<0$ \\
\hline $\begin{array}{c}\text { "vacuum" } \\
\left(\sigma_{\text {halo }}<\sigma_{\text {disk }}\right)\end{array}$ & $\mathrm{A} 0(\mathrm{~A} 1)$ & $\mathrm{S} 1$ & $\mathrm{~S} 1$ \\
$\begin{array}{c}\text { plasma" } \\
\left(\sigma_{\text {halo }}>\sigma_{\text {disk }}\right)\end{array}$ & $\mathrm{S} 0(\mathrm{~S} 1)$ & $\mathrm{A} 0(\mathrm{~A} 1)$ & $\mathrm{A} 1$ \\
\hline
\end{tabular}

axis, $\Omega_{0}$, depends on the turnover radius $r_{\Omega}$. The smaller $r_{\Omega}$, the morc differential rotation we have.

The question is, how effectively the differential rotation inhibits the appearance of non-axisymmetric modes. To this end, we apply a scquence of rotation laws defined with various $r_{\Omega}$ to the A0 and S1 mode from the upper ("vacuum"-)line of Tab. I. The results are shown in Fig. 3. The cigenvalues in $\alpha_{\phi \phi}$ are computed for a set of $r_{\Omega}$. For small $r_{\Omega}$ the $\mathrm{S} 0$-mode is first bifurcating while for larger $r_{\Omega}$ the non-axisymmetry prevails. Both regimes are separated at $r \Omega \simeq 8 \mathrm{kpc}$. If the rigidly rotating core is larger than 8 times the galaxy thickness, the non-axisymmetric S1-mode bifurcates first. Thus we expect very thin disks as good candidates for magnetic BSS-modes. Similar behaviour is shown by the modes for the isotropic $\alpha$-effect. In any case the differential rotation favours the quadrupolar and axisymmetric symmetries. All the special interesting features of the Tab. I are overcome by the dominating differential rotation.

The consideration of radial profiles of the $\alpha$-effect is an interesting extension of the kinematic dynamo theory. So far we have only involved the radius-dependence of the angular velocity which the $\alpha$-effect depends on. Donner \& Brandenburg (1990) have argued that an additional radial profile results from the preferred location of the star formation. They consider both a corc model and a ring model depending on whether the turbulence is also active in the centre. The ring-model is obviously similar to the torus dynamo (Schmitt 1990, Deinzer clal. 1993), reproducing the known S0 mode. The core model, on the other hand, is so close to a spherical $\alpha^{2}$-dynamo that the resultant dipolar mode A0 is no surprise (Elstner el al. 1992).

\section{The angular momentum transport}

Another point of interest is the angular momentum transport by the dynamoexcited magnetic fields. If this transport is outwards then an accretion flow can develop which brings matcrial to the galactic center. Critical for that concept is the 


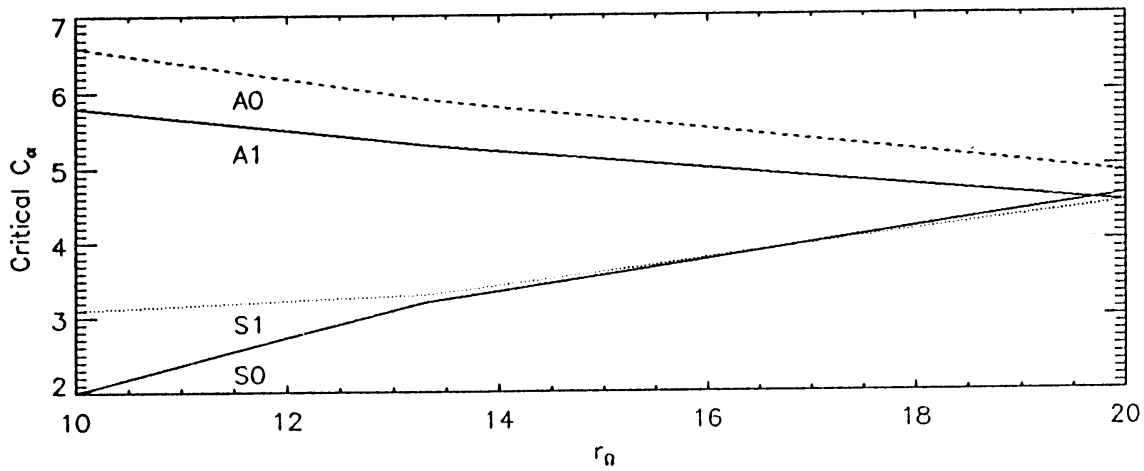

Fig. 3. Critical $\alpha$-cigenvalues for the "vacuum"-models of Tab. I and large or small rigidly-rolating corcs of the galaxy (lincar rotation vclocity $V=100 \mathrm{~km} / \mathrm{s}$ ). Thic radius of the core is given in units of the galaxy thickncss. Note the dominance of the S(-mode

relation of the flow to the local specd of sound. In Rüdiger clal. (1993) it is shown that the local Mach number $\mathcal{M}$ of the accretion flow is

$$
\mathcal{M}=-\frac{r / H}{\mu \rho V^{2} H} \int_{-\infty}^{\infty} \bar{B}_{r} \bar{B}_{\phi} d z .
$$

The Mach number directs the accretion flow:

$$
\dot{m} \simeq 5 H_{1}^{2} V_{100} \mathcal{M} \simeq 1-10 \mathcal{M}
$$

( $H_{1}$ in $\mathrm{kpc}, V_{100}$ in $100 \mathrm{~km} / \mathrm{s}$, in in solar masses per ycar). $\Lambda$ Mach number of order unity would thus allow a remarkable amount of mass accretion - enough to yicld the obscrved quasar brightness.

The dynamo with which we have determined the (maximal) Mach number of the accretion flow opcrated with a rms velocity of $15 \mathrm{~km} / \mathrm{s}$ at the galactic midplane reducing to $8 \mathrm{~km} / \mathrm{s}$ above and below. The halo diflusivily behaves like $(8 / 15)^{2}$ in relation to the central value. In order to study the influence of the hato, however, its (eddy) diffusivity has becn used as a free parancler (cf. Tab. II). F'or increasing conductivity ("plasma") we find an incrcasc in the maximum magnetic field (normalised to the equipartition value at the midplane). Nevertheless the angular momentum transport is more complicaled as it possesses a maximum for medium halo conductivitics. Obviously, there is a complicaled interplay belween magnetic strength, pitch angle and contribution of the halo to the angular momentum transport. In summary, it can be stressed that the absolute amount of the magnetically produced Mach number is, by far, too slow for driving a substantial accretion flow.

\section{The model "U"}

The strength and the geometry of the induced large-scale magnetic fields are the main results in the nonlincar dynamo theory. For the axisymmetric galactic dynamo, in particular, it is the symmetry with respect to the equat,or which must be derived 
TABLE II

The influence of the halo diffusivity on the induced magnetic field strength and the Mach number of the accretion flow $\left(V=200 \mathrm{~km} / \mathrm{s}, r_{\Omega}=2 H\right)$

\begin{tabular}{c|cc}
\hline disk embedded in & $B_{\text {max }} / B_{\text {eq,c }}$ & $10^{4} \mathcal{M}_{\max }$ \\
\hline ("vacuum") & & \\
35 & 2.02 & 6.08 \\
3.5 & 2.03 & 6.44 \\
0.35 & 2.28 & 5.72 \\
0.035 & 3.1 & 5.12 \\
("plasma") & & \\
\hline
\end{tabular}

TABLE III

The influence of the intensity gradient of the interstellar turbulence on the induced magnetic field strength $\left(V=200 \mathrm{~km} / \mathrm{s}, r_{\Omega}=2 H\right)$. Also shown is the parity parameter, $\mathrm{P}$

\begin{tabular}{c|ccccc}
\hline$u_{\max }[\mathrm{km} / \mathrm{s}]$ & 9 & 10 & 15 & 25 & 50 \\
\hline$B_{\max } / B_{\text {eq,c }}$ & 1.9 & 2.3 & 2.4 & 1.9 & 0.4 \\
\hline$B_{\max }[\mu \Gamma]$ & 6 & 8 & 13 & 17 & 7 \\
\hline $\mathrm{P}$ & 1 & 1 & 1 & 1 & -1 \\
\hline & & & & &
\end{tabular}

from the dynamo equation. Quadrupolar modes (S) can occur with dipolar modes (A) - in case of mixing, the parity $\mathrm{P}$ may be used as a good representation of the equatorial symmetry (Brandenburg et al. 1989). The angular momentum transport of only quadrupolar modes allows an accretion flow which is directed inwards.

The magnetic field strength can be measured with respect to the "equipartition field" $B_{e q}=\sqrt{\mu \rho} \sqrt{<u^{\prime 2}>}$. As the turbulence intensity here is non-uniform we have always taken its midplane value.

The model "U" works only with the $\alpha$-effect due to the vertical stratification of the turbulence intensity. The turbulence intensity is assumed to be radiusindependent, which is still a drastic restriction. 


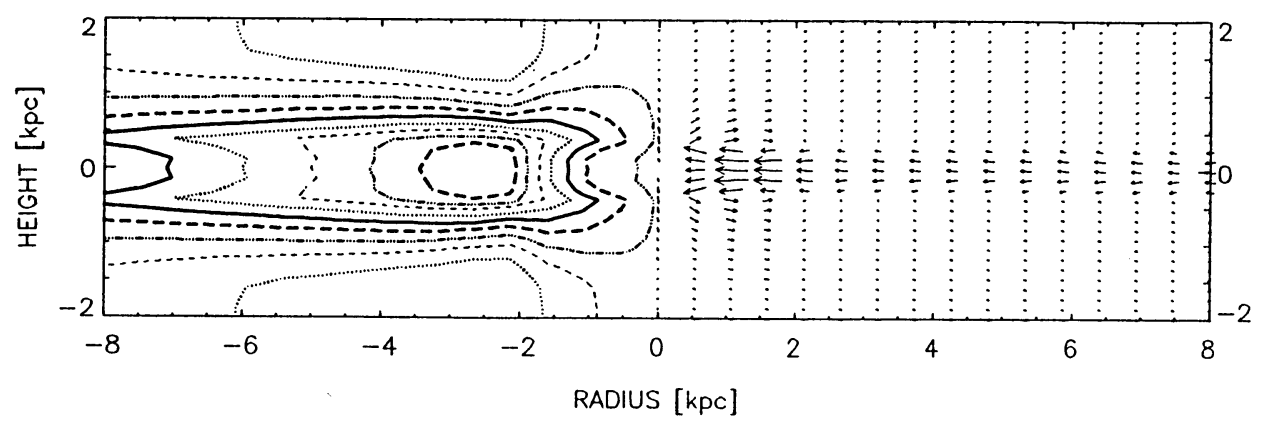

Fig. 4. The dynamo-induced magnetic ficld (left: toroidal, right: poloidal). The radius of the core is given. The inner turbulence rms velocity is $15 \mathrm{~km} / \mathrm{s}$, whilc the outcr velocity is $8 \mathrm{~km} / \mathrm{s}$

The halo value of the rms velocity is fixed to $8 \mathrm{~km} / \mathrm{s}$ while the midplanc value (" $u_{\text {max }} "$ ) is varied as a free parameter. The eddy diffusivity is maximal at the galactic midplane where the $\alpha$-effect vanishes. The $\alpha$-cffect appcars in the region between $0.2-0.4 \mathrm{H}$, where the turbulent diffusivity is smaller. For $z \geq 0.4 \mathrm{H}$ the velocity-profile is again flat hence the $\alpha$-cffect vanishes.

The increase of $u_{\max }$ simultaneously amplifies the eddy diffusivity at the galactic midplane and the $\alpha$-eflect in the $\alpha$-region. The consequences for both the inducing effects ( $\alpha$ and differential rotation) are quite different. For growing $u_{\max }$ it is

$$
C_{\alpha} \simeq \tau_{c o r r} \Omega, \quad C_{\Omega} \simeq \mathcal{M}_{\mathrm{T}^{-2}} \frac{1}{\tau_{\text {corr }} \Omega}
$$

with $\mathcal{M}_{\mathrm{T}}$ as the Mach number of the turbulence at the midplane. For growing $\mathcal{M}_{\mathrm{T}}$ the effect of the differential rotation is basically reduced. The ratio of both inducing effects is

$$
\frac{C_{\alpha}}{C_{\Omega}} \simeq\left(\tau_{c o r r} \Omega\right)^{2} \mathcal{M}_{\mathrm{T}}^{2}
$$

so that for supersonic turbulence, as well as faster rotating galaxics, the differential rotation looses its meaning. The galaxy then changes to an $\alpha^{2}$-dynamo with the properties described in Section 3. Its dynamo number $C_{\alpha}^{2}$ is smaller than the dynamo number of $\alpha \Omega$-dynamos hence the magnetic field strength is reduced. The numbers in Tab. III confirm such statements. We find a maximum of the field strength for $u_{\max } \approx 25 \mathrm{~km} / \mathrm{s}$. For steeper profiles there is indecd indication for a change of the equatorial parity from quadrupoles to dipoles.

For the discussion of the field configuration the model with $u_{\max }=15 \mathrm{~km} / \mathrm{s}$ is selected. From Figs. 4 and 5 we find that the characteristic properties of such models are:

- the fields are confined to the galactic disk,

- the fields have a ringlike structure, 


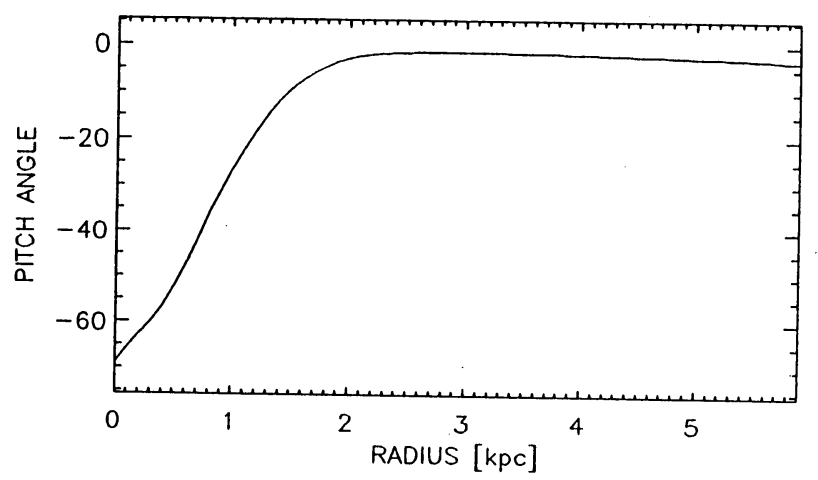

Fig. 5. The pitch angle $\tan \varphi=B_{r} / B_{\phi}$ for the same model as in Fig. 4

- the ring is attached at the turnover radius $r_{\Omega}$,

- the pitch angle is very small except at $r_{\Omega}$.

The agreement with the observations is not perfect. In contrast to the observed characteristics of the magnetic field the modcl is still too close to a pure $\alpha \Omega$-dynamo.

There are several possibilities to overcome the dominating influcnec of the differential rotation. The most powerful one is suggested by Eq. (14). An increasing of the lifetime $\tau_{\text {corr }}$ should amplify the action of the $\alpha$-efrect and also reduce the action of the $\Omega$-effect. (The dynamo number $C_{\alpha} C_{\Omega}$ is not influcnced!) The present computations coinfirm this idea (Fig. 6). For cxample, the pitch angle for a galactic dynamo with $\tau_{\text {corr }}=2 \cdot 10^{7}$ yrs increases to values close to that observed. Thus it is possible to find a lifetime $\tau_{\text {corr }}$, with which the dynamo exhibits the correct values. Only the radial profile of the pitch angle is still in error, i.c. the computed angles grow outwards in opposition to the observations ( $c f$. Beck 1993). We suggest that a variation in the radial profile of the lifetimes helps in resolving this remaining problem.

There is another problem with differential rotation reaching far in to the halo. If the latter consists on plasma with a certain conductivity, the differcntial rotation will induce strong toroidal magnetic belts in the halo which, however, are not observed. This observational fact excludes the cxistence of too high halo conductivities. Low conductivity or even a vacuum prevent the galaxies from becoming solar-type dynamos. The same result could also be reached with rather high turbulent halo velocities (Brandenburg clal. 1993) but in that case one cannot avoid the difficulties with the resulting small or even negative $\alpha$-values in the disk itself.

\section{References}

Beck, R.: 1993, thesc proccedings ,

Brandenburg, A., Tuominen, I., Moss, D.: 1989, Gicophys. Astrophys. Fluid. Dyn. 40,129

Brandenburg, A., Nordlund, A., Pulkkincn, P., Stcin, R.F., Tuonincn, I.: 1990, Astron. Astrophys. 232, 277

Brandenburg, A., Donner, K.J., Moss, D., Shukurov, A.A., Sokoloft, D.D., Tuomincn, I.: 1993, preprint, 


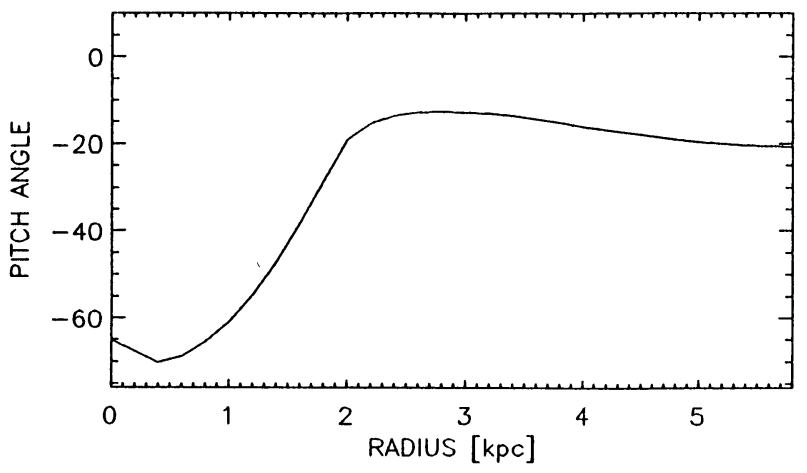

Fig. 6. The pitch angle $\tan \varphi=B_{r} / B_{\phi}$ for the same model as in Fig. 5 but for twice the lifetime $\tau_{c o r r}$

Deinzer, W., Grosser, H., Schmitt, D.: 1993, Astron. Astrophys. , sub.

Donner, K.J., Brandenburg, A.: 1990, Astron. Astrophys. 240, 289

Elstner, D., Meinel, R., Rüdiger, G.: 1990, Geophys. Astrophys. Fluid Dyn. 50, 85

Elstner, D., Meinel, R., Beck, R.: 1992, Astron. Astrophys. Suppl. 94, 587

Ferrière, K.: 1992, Astrophys. J. 389, 286

Ferrière, K.: 1992, Astrophys. J. 391, 188

Gilbert, A.D., Sulem, P.-L.: 1990, Geophys. Astrophys. Fluid Dyn. 51, 243

Kaisig, M., Rüdiger, G., Yorke, H.W.: 1993, Astron. Astrophys. ,

Kichatinov, L.L.: 1987, Geophys. Astrophys. Fluid Dyn. 38, 273

Kichatinov, L.L.: 1991, Astron. Astrophys. 243, 483

Kichatinov, L.L., Rüdiger, G.: 1992, Astron. Astrophys. 260, 494

Krause, F., Meinel, R.: 1988, Geophys. Astrophys. Fluid Dyn. 43, 95

Krause, F., Rädler, K.-H.: 1981, Mean-field magnetohydrodynamics and dynamo theory, Pergamon

Krause, F., Meinel, R., Elstner, D., Rüdiger, G.: 1990, in: R. Beck et al. (eds.): Galactic and Intergalactic Magnetic Fields, IAU No. 140, 97

Krause, M., Beck, R., Hummel, E.: 1989, Astron. Astrophys. 217, 17

Meinel, R., Elstner, D., Rüdiger, G.: 1990, Astron. Astrophys. 236, L33

Moffatt, H.K.: 1970, J. Fluid Mech. 44, 705

Moss, D., Brandenburg, A.: 1992, Astron. Astrophys. 256, 371

Roberts, P.H., Soward, A.: 1975, Astron. Nachrichten 206, 49

Rüdiger, G.: 1980, Astron. Nachrichten 299, 217

Rüdiger, G.: 1990, Geophys. Astrophys. Fluid Dyn. 50, 53

Rüdiger, G., Kichatinov, L.L.: 1993, Astron. Astrophys. 269, 581

Rüdiger, G., Elstner, D.: 1993, Astron. Astrophys. acc.,

Rüdiger, G., Elstner, D., Schultz, M.: 1993, Astron. Astrophys. acc.,

Schmitt, D.: 1990, Reviews in modern astronomy 3, 88

Sofue, Y., Fujimoto, M., Wielebinski, R.: 1988, Ann. Rev. Astron. Astrophys. 24, 459

Steenbeck, M., Krause, F., Rädler, K.-H.: 1966, Z. Naturforsch. 21a, 369

Stepinski, T.F., Levy, E.H.: 1988, Astrophys. J. 331, 416

Wälder, M., Deinzer, W., Stix, M.: 1980, J. Fluid Mech. 96, 207 